\title{
Spontaneously orbital-selective superconductivity in a three-orbital Hubbard model
}

\author{
Kosuke Ishigaki, ${ }^{1}$ Joji Nasu, ${ }^{1}$ Akihisa Koga, ${ }^{1}$ Shintaro Hoshino, ${ }^{2}$ and Philipp Werner ${ }^{3}$ \\ ${ }^{1}$ Department of Physics, Tokyo Institute of Technology, Meguro, Tokyo 152-8551, Japan \\ ${ }^{2}$ Department of Physics, Saitama University, Saitama 338-8570, Japan \\ ${ }^{3}$ Department of Physics, University of Fribourg, 1700 Fribourg, Switzerland
}

(Received 1 June 2018; published 10 December 2018)

\begin{abstract}
We study a three-orbital Hubbard model with negative Hund's coupling in infinite dimensions, combining dynamical mean-field theory with continuous time quantum Monte Carlo simulations. This model, which is relevant for the description of alkali-doped fullerides, has previously been shown to exhibit a spontaneous orbital-selective Mott phase in the vicinity of the superconducting phase. Calculating the pair potential and double occupancy in each orbital, we study the competition between different homogeneous ordered states and determine the corresponding finite-temperature phase diagram of the model. We identify two distinct types of spontaneous orbital-selective Mott states and show that an orbital-selective $s$-wave superconducting state with one superconducting and two metallic orbitals is spontaneously realized between the conventional $s$-wave superconducting phase and these two kinds of spontaneously orbital-selective Mott states.
\end{abstract}

DOI: 10.1103/PhysRevB.98.235120

\section{INTRODUCTION}

Orbital degrees of freedom and their dynamics are known to play an essential role in strongly correlated electron systems as they couple to other degrees of freedom of the lattice system. This can lead to exotic phenomena such as colossal magnetoresistance in the manganites [1] and unconventional superconductivity in ruthenates [2] or iron pnictides [3]. An interesting phenomenon in this general context is the orbitalselective Mott (OSM) transition [4], which has been discussed in transition-metal oxides such as $\mathrm{Ca}_{2-x} \mathrm{Sr}_{x} \mathrm{RuO}_{4}[5,6]$ and $\mathrm{La}_{n+1} \mathrm{Ni}_{n} \mathrm{O}_{3 n+1}$ [7-9]. The OSM transition results in a distinct electronic character of different orbitals; that is, some orbitals are itinerant, while the others are localized. This physics has been explored in simple two-orbital Hubbard models with different bandwidths [10-14] or crystal field splittings [15], where the difference in the effective Coulomb interaction or local energy induces the OSM state.

Orbital-selective physics in a model with degenerate bands is less trivial since it corresponds to a spontaneous breaking of symmetry, and an interesting question is whether such an OSM transition occurs simultaneously with a spontaneous orbital order. Recently, an exotic state with itinerant and localized orbitals has indeed been observed in the fullerenebased solids $A_{3} \mathrm{C}_{60}(A=$ alkali metal $)$ with triply degenerate $t_{1 u}$ orbitals [16], which motivates further theoretical and experimental investigations on orbital-selective phenomena in such multiorbital systems. A previous study of a half-filled three-orbital Hubbard model with antiferromagnetic Hund's coupling [17] revealed the existence of an OSM state with spontaneously broken orbital symmetry (two Mott insulating and one metallic orbitals), and this state has been referred to as a spontaneously orbital-selective Mott (SOSM) state. Furthermore, it was demonstrated that this SOSM state is realized in the vicinity of an $s$-wave superconducting (SC) dome and a Mott insulating phase, which is consistent with the phase diagram of fullerene-based solids [16]. However, these insights were based on susceptibility calculations in the symmetric phase, and the competition between the SC and SOSM states and also the role of orbital fluctuations at low temperature were not addressed. To clarify these issues, it is important to directly examine the symmetry-broken states.

In this paper, we study the three-orbital Hubbard model with antiferromagnetic Hund's coupling at half filling, combining dynamical mean-field theory (DMFT) [18-20] with continuous-time quantum Monte Carlo (CTQMC) simulations $[21,22]$. Calculating pair potentials and double occupancies, we clarify that at low temperatures, an $s$-wave SC state without orbital symmetry breaking is stabilized rather than the SOSM state. At higher temperatures, we find a new SOSM state for which two orbitals are metallic, while the third is in a paired Mott state. Most remarkably, we demonstrate that this SOSM phase transforms into a spontaneous orbital-selective superconducting (SOSSC) phase in the vicinity of the SC dome.

This paper is organized as follows. In Sec. II, we introduce the model Hamiltonian and briefly summarize our numerical method. In Sec. III, calculating various physical quantities, we clarify how orbital-selective phases are spontaneously realized in the three-orbital Hubbard model. Then, we determine the finite-temperature phase diagram. A summary is given in the final section. In the Appendix, the effect of the exchange and pair hopping is discussed in terms of a phenomenological theory.

\section{MODEL AND METHOD}

We consider the half-filled three-orbital Hubbard model described by the Hamiltonian

$$
\begin{aligned}
\mathcal{H}= & -t \sum_{\langle i, j\rangle \alpha \sigma} c_{i \alpha \sigma}^{\dagger} c_{j \alpha \sigma}+U \sum_{i \alpha} n_{i \alpha \uparrow} n_{i \alpha \downarrow} \\
& +U^{\prime} \sum_{i \sigma \alpha<\beta} n_{i \alpha \sigma} n_{i \beta \bar{\sigma}}+\left(U^{\prime}-J\right) \sum_{i \sigma \alpha<\beta} n_{i \alpha \sigma} n_{i \beta \sigma},
\end{aligned}
$$


where $c_{i \alpha \sigma}$ is the annihilation operator for an electron with spin $\sigma(\uparrow, \downarrow)$ and orbital index $\alpha(=1,2,3)$ at the $i$ th site and $n_{i \alpha \sigma}=c_{i \alpha \sigma}^{\dagger} c_{i \alpha \sigma} ; t$ is the transfer integral between nearestneighbor sites, $U\left(U^{\prime}\right)$ is the intraband (interband) Coulomb interaction, and $J$ is Hund's coupling. We assume the relation $U=U^{\prime}+2 J$ since it approximately holds even in the fullerides, where the intraorbital $U$, interorbital $U^{\prime}$, and Hund's coupling $J$ are renormalized by the coupling to Jahn-Teller phonons [23]. For simplicity, we neglect the exchange part of Hund's coupling and the pair-hopping term. The effects of these interactions are discussed later.

In the present calculations, we fix Hund's coupling as $J / U=-1 / 4$, which is large compared to $a b$ initio estimates [24] but allows us to reveal the relevant physics at moderate computational expense. An important point is the negative sign of the (antiferromagnetic) coupling, which is characteristic of fullerene-based solids [25-27]. This coupling disfavors singly occupied orbitals since the interorbital Coulomb interaction dominates $\left(U^{\prime}>U\right)$. At half filling, the intraorbital Coulomb interaction can be regarded as effectively attractive in the weak-coupling region. On the other hand, in the strongly interacting half-filled case, empty and singly and doubly occupied orbitals are realized on a given site, and hence, large orbital fluctuations are expected in the system. This is in stark contrast to the ferromagnetic Hund's coupling case with $U>U^{\prime}$, where each orbital wants to be singly occupied and orbital fluctuations are suppressed. Therefore, in our model with antiferromagnetic Hund's coupling $(J<0)$, interesting orbital-selective states may emerge due to orbital fluctuations.

In the present study, we mainly present results based on DMFT. In this approach, the lattice model is mapped to an effective impurity problem, where local electron correlations can be taken into account precisely. The Hubbard model with degenerate orbitals has been extensively discussed in the framework of DMFT, and interesting phenomena have successfully been clarified such as the Mott transition [2835], orbital-selective Mott transitions [10-15,36], magnetism [37-39], and superconductivity [17,40,41]. In DMFT, the lattice enters via the self-consistency conditions imposed on the impurity problem. This treatment is exact in $d \rightarrow \infty$ dimensions, and even in three dimensions, reasonable results have been obtained [17]. When the superconducting state is considered in this framework, the Green's functions are represented in the Nambu formalism. The impurity Green's functions for orbital $\alpha$ are given by

$$
\hat{G}_{\mathrm{imp}, \alpha}(\tau)=\left(\begin{array}{cc}
G_{\alpha \uparrow}(\tau) & F_{\alpha}(\tau) \\
F_{\alpha}^{*}(\tau) & -G_{\alpha \downarrow}(-\tau)
\end{array}\right),
$$

where the normal and anomalous Green's functions are defined as

$$
\begin{aligned}
G_{\alpha \sigma}(\tau) & =-\left\langle T_{\tau}\left[c_{\alpha \sigma}(\tau) c_{\alpha \sigma}^{\dagger}\right]\right\rangle, \\
F_{\alpha}(\tau) & =-\left\langle T_{\tau}\left[c_{\alpha \uparrow}(\tau) c_{\alpha \downarrow}\right]\right\rangle
\end{aligned}
$$

and $T_{\tau}$ is the imaginary-time ordering operator. In our calculation, we use a semicircular density of states, $\rho(x)=$ $2 /(\pi D) \sqrt{1-(x / D)^{2}}$, where $D$ is the half bandwidth. The self-consistency equation [42] is given by

$$
\hat{\mathcal{G}}_{\alpha}(z)=z \hat{\sigma}_{0}+\mu \hat{\sigma}_{z}-\frac{D^{2}}{4} \hat{\sigma}_{z} \hat{G}_{\mathrm{imp}, \alpha}(z) \hat{\sigma}_{z},
$$

where $\hat{\sigma}_{0}$ is the identity matrix and $\hat{\sigma}_{z}$ is the $z$ component of the Pauli matrix. $\hat{\mathcal{G}}\left(\hat{G}_{\text {imp }}\right)$ is the noninteracting (full) Green's function for the effective impurity model. In our study, we use, as an impurity solver, the hybridization-expansion CTQMC algorithm [21,22], which is one of the most powerful methods to discuss finite-temperature properties in multiorbital models.

\section{NUMERICAL RESULTS}

In the present study, we focus on the half-filled model. In the fcc fullerides, the antiferromagnetic phase transition temperature $\left(T_{N} \simeq 2 \mathrm{~K}\right)$ is significantly suppressed compared to the bcc fullerides $\left(T_{N} \simeq 40 \mathrm{~K}\right)$ due to the effect of geometrical frustration [43]. With this class of materials in mind, we assume the suppression of antiferromagnetic (and also antiferro-orbital) orders and aim at a simplified model description of the relatively high temperature regime.

To examine the competition between the SC and SOSM states we calculate the pair potential in the $\alpha$ th orbital,

$$
\psi_{\alpha}=\left|\left\langle c_{i \alpha \uparrow} c_{i \alpha \downarrow}\right\rangle\right|,
$$

as the order parameter of the SC state. In contrast, the order parameter for the SOSM states is not obvious since no difference in the average orbital occupations appears [17]. Here, we calculate the double occupancy for orbital $\alpha$,

$$
d_{\alpha}=\left\langle n_{i \alpha \uparrow} n_{i \alpha \downarrow}\right\rangle,
$$

and characterize the SOSM state by the appearance of orbitaldependent double occupancies. In the following, we set the unit of energy to the half bandwidth $D$.

Figure 1 plots the pair potential and double occupancy for each orbital at $T / D=0.01$. In the noninteracting case $(U=$ 0 ), a metallic state is realized with $d_{\alpha}=1 / 4$ and $\psi_{\alpha}=0$ for all orbitals. Turning on the interaction $U$ slightly increases the double occupancy since the on-site interaction in this half-filled system is effectively attractive due to the antiferromagnetic Hund's coupling. This interaction is expected to enhance pair correlations, and indeed, a second-order phase transition occurs to the $s$-wave SC state with finite $\psi_{1}=\psi_{2}=$ $\psi_{3}$ around $U / D \sim 0.87$, as shown in Fig. 1(b).

A further increase in the Coulomb interaction leads to a maximum in the pair potentials near $U / D \approx 1.4$. Around $U / D \sim 2.0$, the physical quantities jump, and a first-order phase transition occurs, as shown in Figs. 1(a) and 1(b). In the state with $2.0 \lesssim U / D \lesssim 2.8$, the pair potentials vanish, while the double occupancies take two distinct values, $d_{1}<$ $d_{2}=d_{3}$. This means that a metallic state is realized in orbital 1. In contrast, a paired Mott state with $d \sim 0.5$ is realized in orbitals 2 and 3, which are dominated by empty and doubly occupied configurations. From these observations, we can conclude that an SOSM state is indeed realized in this region [17]. A similar orbital symmetry breaking has also been identified in the two-dimensional system [44]. Beyond $U / D \sim 2.8$, the orbital-selective features disappear, and the double occupancies exhibit an orbital-independent value. In 

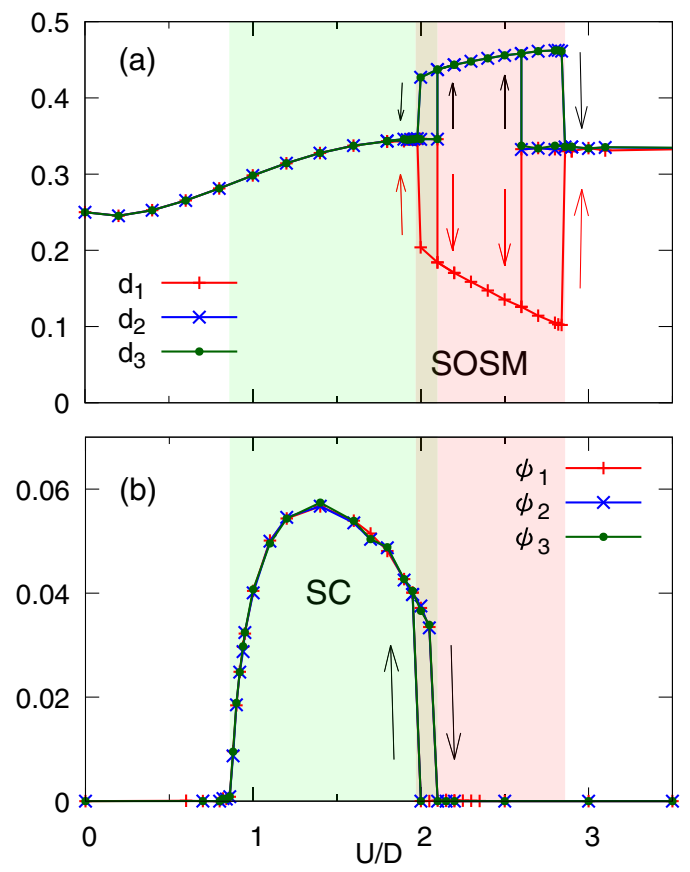

FIG. 1. (a) Double occupancy and (b) pair potential as a function of the interaction strength $U$ in the three-orbital Hubbard model at $T / D=0.01$. The arrows indicate the existence of a hysteresis in these quantities.

the strong-coupling region, three electrons are localized at each site in a configuration with empty, singly, and doubly occupied orbitals, and a Mott state is realized with $d_{\alpha} \sim 1 / 3$.

These results confirm that the SOSM state with one orbital itinerant and two orbitals localized competes with the $s$-wave SC and Mott states at low temperatures. On the other hand, it is naively expected that another SOSM state with two orbitals itinerant and one orbital paired may also exist in the present system, although such a state has not been previously discussed [17]. To clarify this, we examine the low-temperature properties by calculating the orbitaldependent quantities $Z_{\alpha}=\left[1-\operatorname{Im} \Sigma_{\alpha}\left(i \omega_{0}\right) / \omega_{0}\right]^{-1}$ and $A_{\alpha}=$ $-G_{\alpha}(1 / 2 T) / \pi T$, where $\Sigma_{\alpha}$ is the self-energy for the $\alpha$ th orbital and $\omega_{0}=\pi T$. At low temperature, $Z_{\alpha}\left(A_{\alpha}\right)$ yields a good estimate of the renormalization factor (density of states at the Fermi level) [45], so that these quantities allow us to discuss the correlation effects in the metallic state. More specifically, large (small) values of $Z_{\alpha}$ and $A_{\alpha}$ indicate a metallic (insulating) behavior of the $\alpha$ th orbital. By focusing on the orbital differentiation, we can detect and characterize different types of SOSM states. The results at the temperature $T / D=0.02$ are shown in Figs. 2(a)-2(c). In this parameter region, no pair potentials appear. It is found that two kinds of SOSM states are realized between the metallic and Mott states. These can be classified by the number of itinerant unpaired orbitals; the SOSM- $n$ state is associated with $n$ $(=1,2)$ metallic orbital(s), which are schematically shown in Fig. 2(d). When $2.2 \lesssim U / D \lesssim 2.5, d_{1}<d_{2}=d_{3}$, and the SOSM-1 state is realized with a metallic orbital 1 . On the other hand, in the region $2.1 \lesssim U / D \lesssim 2.3, d_{1}=d_{2}<d_{3}$, and the SOSM-2 state is realized with metallic orbitals 1 and 2. The phase transitions between metallic, SOSM-2, SOSM-1,
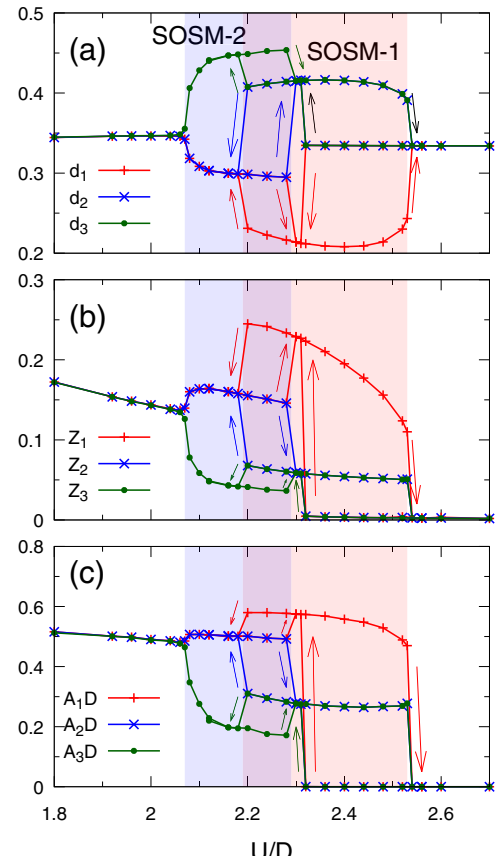

(d)

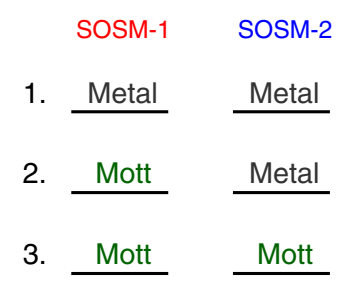

$d_{1}<d_{2}=d_{3} \quad d_{1}=d_{2}<d_{3}$

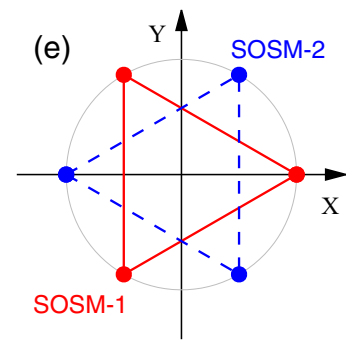

FIG. 2. (a) Double occupancy $d_{\alpha}$, (b) renormalization factor $Z_{\alpha}$, and (c) the quantity $A_{\alpha}$ as a function of $U / D$ when $T / D=0.02$. The arrows indicate the existence of hysteresis in these quantities. (d) Schematic pictures of the SOSM-1 and SOSM-2 states. (e) Classification of orbital orders in the three-orbital Hubbard model. The points connected by lines indicate the equivalent solutions.

and Mott states should be of first order, although no hysteresis is visible around $U / D \sim 2.1$.

To clarify the nature of the phase transition, we employ a Landau theory, where the symmetry of the system is taken into account correctly. As discussed above, the orbital-dependent double occupancies $d_{\alpha}$ are appropriate to characterize the SOSM states [17]. The Landau free energy $F$ can be expanded in powers of $d_{\alpha=1,2,3}$ while taking into account the permutation symmetries among $\gamma=1,2,3$ and the equivalence between $\sigma=\uparrow, \downarrow$. A detailed derivation of the free energy involving Gell-Mann matrices can be found in the Supplemental Material of Ref. [17]. The final result can be expressed as

$$
F=F_{0}+a\left(X^{2}+Y^{2}\right)+b X\left(X^{2}-3 Y^{2}\right)+c\left(X^{2}+Y^{2}\right)^{2},
$$

where $X=\left(d_{1}+d_{2}-2 d_{3}\right) / \sqrt{3}, Y=d_{1}-d_{2}$, with constants $F_{0}, a, b$, and $c(>0) . X$ and $Y$ correspond to the order parameters characteristic of the SOSM states, and their forms derive from the $3 \times 3$ Gell-Mann matrices $\lambda_{8}$ and $\lambda_{3}$, respectively. The orbital permutation is then represented by the $C_{3 V}$ symmetry in the $X-Y$ plane. This yields the thirdorder term in the free energy, and the phase transition to the SOSM states is of first order (related to the Lifshitz condition).

The nontrivial solutions can be classified into two classes. The solution with $(X, Y)=\left(-\frac{1}{2} R,-\frac{\sqrt{3}}{2} R\right)(R>0$ is a radius), which is equivalent to $(R, 0)$ and $\left(-\frac{1}{2} R, \frac{\sqrt{3}}{2} R\right)$ under the $C_{3 V}$ symmetry, corresponds to the SOSM-1 state with $d_{1}<d_{2}=d_{3}$. The other class is the SOSM- 2 solution with 

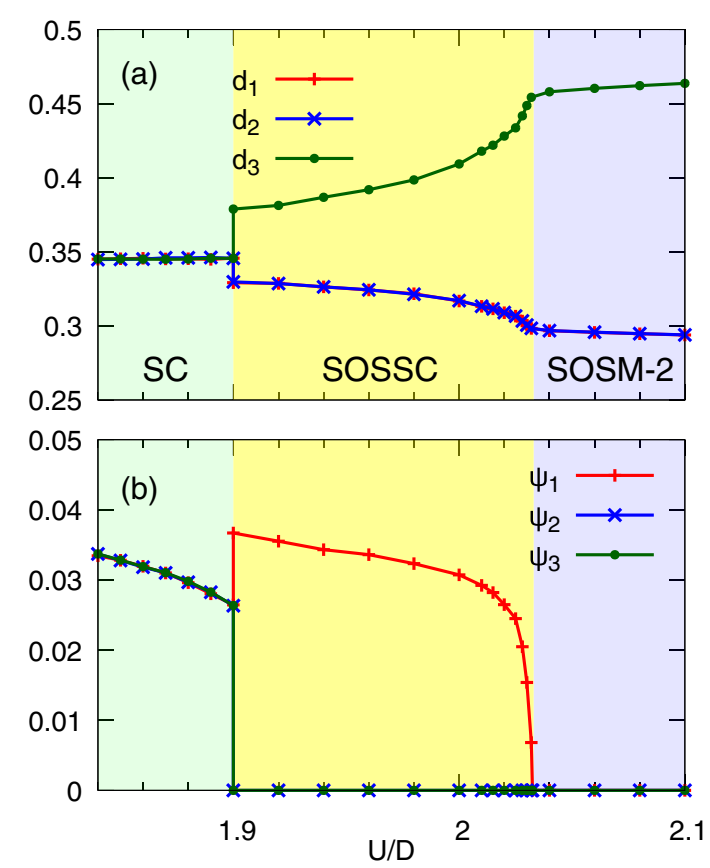

FIG. 3. (a) Pair potential and (b) double occupancy at $T / D=$ 0.013 .

$(X, Y)=(-R, 0)$, where $d_{1}=d_{2}<d_{3}$. These solutions in the $X-Y$ plane are schematically shown in Fig. 2(e). Namely, the SOSM-1 (SOSM-2) state is stabilized in the negative (positive) $b$ case. Therefore, in the system at $T / D=0.02$, the sign of $b$ changes around $U / D \sim 2.25$.

At lower temperatures, an interesting orbital-selective state appears. Figure 3 shows the double occupancy and pair potential for each orbital when $T / D=0.013$. When $U / D \lesssim 1.90$, the $s$-wave SC state is realized with $\psi_{1}=\psi_{2}=\psi_{3} \neq 0$. In the region with $U / D \gtrsim 2.03$, the SOSM-2 state is realized with $d_{1}=d_{2}<d_{3}$ and $\psi_{1}=\psi_{2}=\psi_{3}=0$. Between these two states $(1.90 \lesssim U / D \lesssim 2.03)$, we find in Fig. 3 that the pair potentials as well as the double occupancies take two distinct values. In particular, one of the three orbitals has a finite pair potential, while it vanishes for the other two. This implies the realization of a SOSSC state.

Now, let us consider the nature of the SOSSC state. As shown in Fig. 3, the phase transition at $U / D \sim 1.90$ is of first order, whereas that at $U / D \sim 2.03$ appears to be continuous. This suggests that the SOSSC state is closely related to the higher $U$ state, i.e., the SOSM-2 state. In this state, orbital 3 is in a paired Mott state with large $d_{3}$, and the others are metallic. Decreasing $U$ from the SOSM-2 phase, $\psi_{3}$ becomes finite at $U / D \sim 2.03$, with an accompanying rapid decrease of the double occupancy $d_{3}$. This indicates that orbital 3 plays an essential role in the phase transition to the SOSSC state. The behavior observed in orbital 3 is similar to the lowtemperature properties of the single-band attractive Hubbard model at half filling, where a second-order phase transition occurs between the SC and paired Mott states [46,47]. This is consistent with the present result that the phase transition at $U / D \sim 2.03$ is of second order.

The orbital-selective superconducting instability originates from the existence of paired Mott orbitals in the SOSM

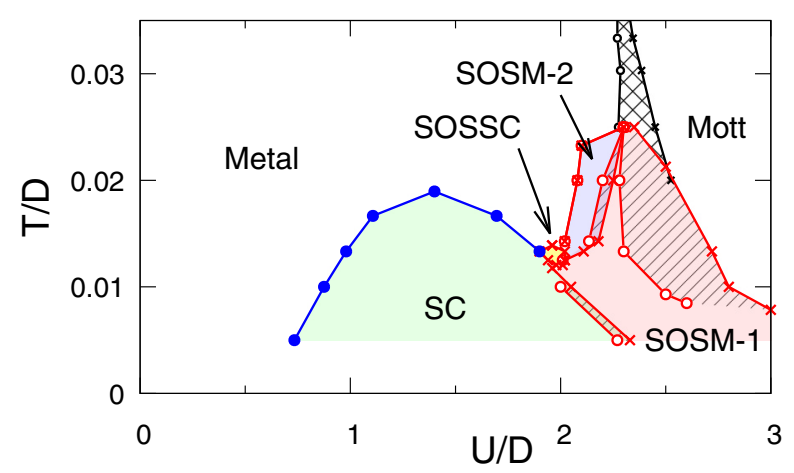

FIG. 4. Phase diagram of the three-orbital Hubbard model. Solid circles represent second-order phase transition points. Open circles (crosses) represent the transition points, where the strong- (weak-) coupling state disappears. Shaded areas bounded by these points indicate the regions with two competing solutions.

state. Therefore, a different type of SOSSC state may exist adjacent to the SOSM-1 state, whose essential feature should be described in terms of the two-band Hubbard model. The SC state in the latter model is realized in a narrow parameter space [40], which suggests that the potential SOSSC state related to the SOSM-1 phase is less stable and is difficult to realize in the present parameter regime.

By performing similar calculations for different temperatures, we obtain the phase diagram shown in Fig. 4, which clarifies the competition between the different ordered phases. For example, this phase diagram indicates that the first-order phase boundary between the $s$-wave SC and SOSM- 1 states shifts to larger $U$ with decreasing temperature. In the strongercoupling region and at low temperatures, the SOSM-1 state is realized instead of the Mott state adjacent to the SC state. This means that, due to the breaking of orbital symmetry, the SOSM-1 state is stabler than the Mott state.

The dominant electronic configurations for the SOSM-1 and SOSM-2 states are similar, but the SOSM-2 state is stabilized only at higher temperatures. This can be explained as follows. The SOSM states possess both itinerant and paired Mott orbitals. The entropies for itinerant and paired Mott orbitals should be given by $S \sim \gamma T$ and $S \sim \ln 2$, respectively, where $\gamma$ is the specific heat coefficient proportional to the effective mass. Therefore, at high temperatures, the metallic orbitals tend to have a large entropy. For this reason, the SOSM-2 state with two metallic orbitals is realizable only at intermediate temperatures. For similar reasons, the SOSSC state with one orbital superconducting and the others metallic is less stable than the SC state with all orbitals superconducting at zero temperature. Therefore, the SOSSC state is stabilized only at nonzero temperatures.

Let us briefly discuss the effect of Hund's coupling. Figure 5 shows the phase diagram of the three-orbital Hubbard model at the fixed temperature $T / D=0.013$. It is found that by increasing $|J / U|$ at fixed $U / D$, metallic, SC, SOSSC, and SOSM-2 states are realized. For the $U / D$ values considered, the SOSSC state exists in the range $0.2 \lesssim|J / U| \lesssim 0.25$. At a somewhat smaller value of $|J / U|$ than used in Fig. 4, the SOSSC phase in the $T-U$ phase diagram would be considerably enhanced. We note, however, that the realistic values 


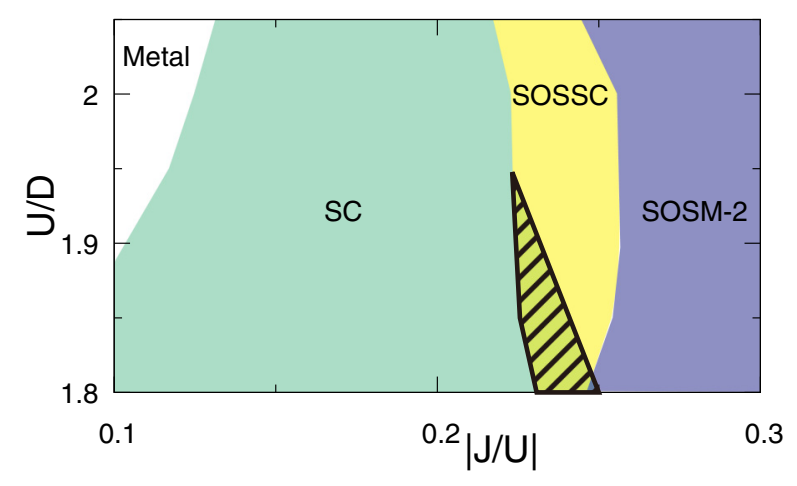

FIG. 5. Phase diagram of the three-orbital Hubbard model at $T / D=0.013$. The hashed area indicates a region with competing solutions.

of $|J / U|$ in alkali-doped fullerides are of the order of 0.02 [27]. On the other hand, in the present DMFT calculations, we did not consider the spin exchange part of Hund's coupling and the pair-hopping term. For the system with antiferromagnetic Hund's coupling, the low-spin state is favored, and the spin flip is irrelevant. It was clarified in Ref. [17] that the pair hopping substantially stabilizes the SOSM-1 and SC states and that the SOSM-1 state can be realized even for a small $|J|$.

To reveal the effect of the pair hopping on the SOSM2 state, we adopt a phenomenological theory (see the Appendix). In the case without pair hopping, the SOSM-2 state appears between the high-temperature metallic and lowtemperature SOSM-1 states, which is consistent with the result obtained by the DMFT calculations. This supports the validity of our phenomenological theory for the present system. Applying it to the system with pair hopping yields the prediction that the SOSM-2 state also exists in the intermediatetemperature region. This result suggests that the SOSM-2 state survives even in the presence of pair hopping. An explicit DMFT study of the SOSM-2 and SOSSC phase boundary in the presence of pair hopping is computationally expensive and beyond the scope of the present study.

\section{SUMMARY}

In summary, we have studied the three-orbital Hubbard model with negative Hund's coupling in infinite dimensions, combining DMFT with the CTQMC method. Calculating the pair potential and double occupancy in each orbital, we have determined the finite-temperature phase diagram of the model with density-density interactions. We have clarified that an orbital-selective $s$-wave superconducting state with one orbital superconducting and the others metallic is spontaneously realized in a small parameter region, in addition to the conventional $s$-wave superconducting state and two kinds of spontaneously orbital-selective Mott states.

A relevant question is if some of the phases revealed in this study are realized in real materials. The Jahn-Teller metal in the fullerene-based solids $A_{3} \mathrm{C}_{60}$ is a promising candidate for the SOSM-1 state [17]. Our results suggest that the higher-temperature part of the Jahn-Teller metal is, in fact, an SOSM-2 state. If one can experimentally distinguish the SOSM-2 state from the low-temperature SOSM-1 state in fcc-type $A_{3} \mathrm{C}_{60}$, for example, by a difference in the electrical conductivity, it may be worthwhile to search for signatures of the spontaneous orbital-selective superconducting state in the vicinity of this transition line and the SC phase. However, because of the small value of $|J / U|$ in fulleride compounds, the SOSSC may not be stable. A quantitative treatment with additional and realistic parameters, including longer-range hoppings, intersite interactions, etc., is necessary to discuss the low-temperature phase diagram of specific compounds. This is an interesting topic for future studies.

\section{ACKNOWLEDGMENTS}

Parts of the numerical calculations were performed in the supercomputing systems in ISSP, the University of Tokyo. This work was supported by a Grant-in-Aid for Scientific Research from JSPS, KAKENHI Grants No. JP18K04678, No. JP17K05536 (A.K.), No. JP16K17747, No. JP16H02206, No. JP18H04223 (J.N.), and No. JP16H04021 (S.H.), and the European Research Council through ERC Consolidator Grant No. 724103 (P.W.). The simulations were performed using some of the ALPS libraries [48].

\section{APPENDIX: PHENOMENOLOGICAL THEORY}

Here, we develop a phenomenological theory that accounts for the thermodynamics of SOSM states. We introduce the labels 0,1 , and 2 to indicate the orbital-symmetric metal state (three metallic orbitals), the SOSM-1 state (one metallic orbital and two paired orbitals), and the SOSM-2 state (two metallic orbitals and one paired orbital), respectively. A metallic orbital results in a free-energy gain from the kinetic energy $K$ and the $T$-linear entropy ( $S \propto T)$. For a paired orbital, on the other hand, there is a free-energy gain from the effective attraction $V$ and also from the entropy $S=\ln 2$ associated with the degrees of freedom of locating the pairs in the orbitals. The effective free energies can thus be expressed as

$$
\begin{gathered}
F_{0}=-3 K-T(3 \gamma T), \\
F_{1}=-K-2 V-T(\ln 2+\gamma T), \\
F_{2}=-2 K-V-T(\ln 2+2 \gamma T),
\end{gathered}
$$

where $K>0, V>0, \gamma>0$ are the (renormalized) kinetic energy, effective attraction, and specific heat coefficient $(\gamma \sim 1 / K)$, respectively. Our purpose here is to describe the thermodynamic stability of these states, while the kinetic energy of the pairs, which is necessary for, e.g., superconductivity, is neglected. We focus on the low-energy region and neglect the $T$ dependence of $K, V, \gamma$.

We are interested in the stability of the SOSM- 2 state on which the SOSSC is founded. The inequalities $F_{0}>F_{2}$ and $F_{1}>F_{2}$ are the necessary and sufficient conditions for the realization of SOSM-2 as the stablest state. This leads to the relation

$$
V>K-\frac{(\ln 2)^{2}}{4 \gamma}
$$


Without pair hopping, $\mathrm{V}=0$

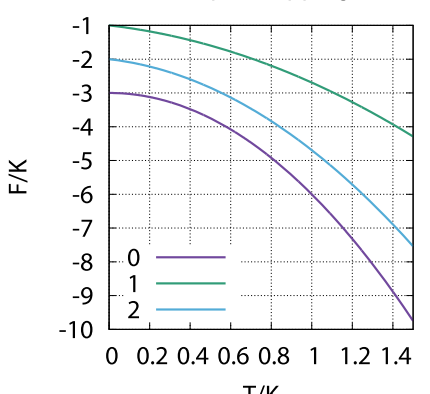

$\mathrm{T} / \mathrm{K}$

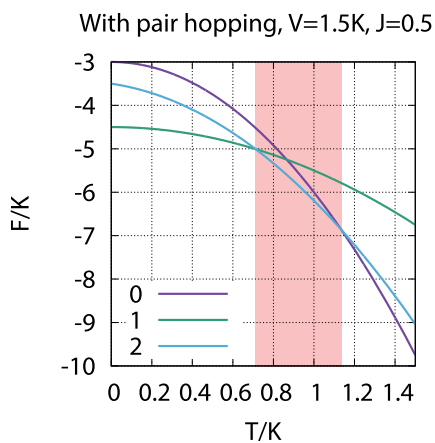

Without pair hopping, $\mathrm{V}=0.9 \mathrm{~K}$

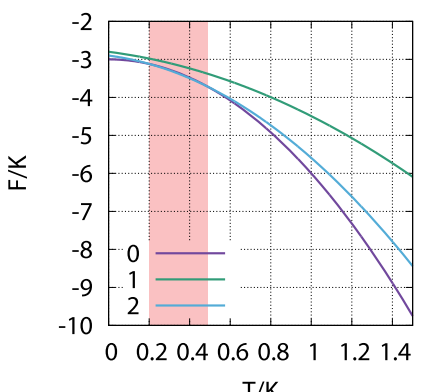

$\mathrm{T} / \mathrm{K}$

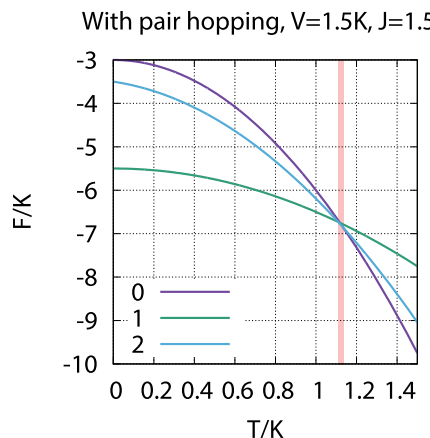

Without pair hopping, $\mathrm{V}=1.5 \mathrm{~K}$

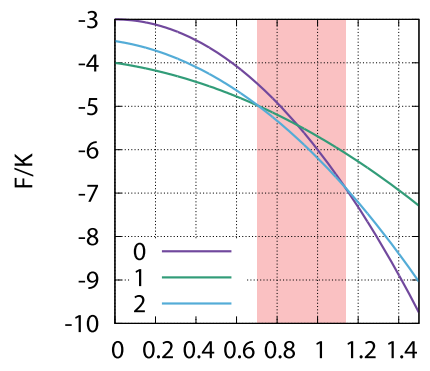

$\mathrm{T} / \mathrm{K}$
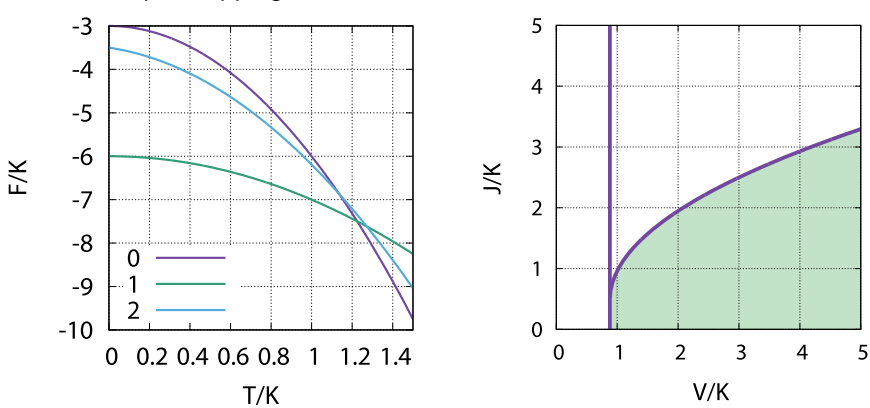

FIG. 6. Temperature dependences of the free energies without (top panels) and with (bottom panels) pair hopping. We have used $\gamma=\alpha / K$ with $\alpha=1$. The intervals highlighted in red indicate the temperature range where the SOSM-2 is the most stable. The rightmost panel in the bottom row shows the stability region of the SOSM-2 state determined by Eqs. (A8) and (A9).

which determines the lower bound of the attractive interaction $V$. The typical temperature dependences of the free energy are shown in the top panels of Fig. 6. When the effective attraction is sufficiently large, the SOSM-2 state becomes the stablest one in the intermediate-temperature range, which is qualitatively consistent with the results shown in the main text. This demonstrates that the above simple theory can capture the thermodynamics of the SOSM states realized in the DMFT study.

Since the above phenomenology works well for the effective description of the DMFT results, we now apply it to the system with pair hopping. The free energies in this case are

$$
\begin{gathered}
F_{0}=-3 K-T(3 \gamma T), \\
F_{1}=-K-2 V-J-T(\gamma T), \\
F_{2}=-2 K-V-T(\ln 2+2 \gamma T),
\end{gathered}
$$

where $J>0$ is the (effective) pair hopping. The pair hopping modifies only $F_{1}$ since the two paired orbitals are quantummechanically mixed, which results in an energy gain of $J$. The condition for the situation where SOSM-2 is stablest in the presence of pair hopping becomes

$$
\begin{gathered}
V>K-\frac{(\ln 2)^{2}}{4 \gamma}, \\
J<\frac{\ln 2}{\gamma}\left[\ln 2+\sqrt{(\ln 2)^{2}+4 \gamma(V-K)}\right] .
\end{gathered}
$$

Thus, there is an upper bound for the magnitude of $J$ in addition to a lower bound for $V$. The typical temperature dependences of the free energies are shown in the bottom panels of Fig. 6, and the necessary conditions described by Eqs. (A8) and (A9) are indicated in the rightmost panel. Although it is not trivial to determine whether or not the realistic values are located inside of this region, there is a chance that the SOSM-2 state is realized if the effective attraction $V$ is strong enough, which pushes the upper bound of $J$ to higher values.
[1] Y. Tokura, A. Urushibara, Y. Moritomo, T. Arima, A. Asamitsu, G. Kido, and N. Furukawa, J. Phys. Soc. Jpn. 63, 3931 (1994).

[2] Y. Maeno, M. Rice, and M. Sigrist, Phys. Today 54(1), 42 (2001).

[3] Y. Kamihara, H. Hiramatsu, M. Hirano, R. Kawamura, H. Yanagi, T. Kamiya, and H. Hosono, J. Am. Chem. Soc. 128, 10012 (2006).

[4] V. I. Anisimov, I. A. Nekrasov, D. E. Kondakov, T. M. Rice, and M. Sigrist, Eur. Phys. J. B 25, 191 (2002).
[5] S. Nakatsuji and Y. Maeno, Phys. Rev. Lett. 84, 2666 (2000).

[6] S. Nakatsuji, D. Hall, L. Balicas, Z. Fisk, K. Sugahara, M. Yoshioka, and Y. Maeno, Phys. Rev. Lett. 90, 137202 (2003).

[7] K. Sreedhar, M. McElfresh, D. Perry, D. Kim, P. Metcalf, and J. Honig, J. Solid State Chem. 110, 208 (1994).

[8] Z. Zhang and M. Greenblatt, J. Solid State Chem. 117, 236 (1995).

[9] Y. Kobayashi, S. Taniguchi, M. Kasai, M. Sato, T. Nishioka, and M. Kontani, J. Phys. Soc. Jpn. 65, 3978 (1996). 
[10] A. Koga, N. Kawakami, T. M. Rice, and M. Sigrist, Phys. Rev. Lett. 92, 216402 (2004).

[11] K. Inaba, A. Koga, S.-I. Suga, and N. Kawakami, J. Phys. Soc. Jpn. 74, 2393 (2005).

[12] A. Koga, N. Kawakami, T. M. Rice, and M. Sigrist, Phys. Rev. B 72, 045128 (2005).

[13] K. Inaba and A. Koga, Phys. Rev. B 73, 155106 (2006).

[14] L. de' Medici, S. R. Hassan, M. Capone, and X. Dai, Phys. Rev. Lett. 102, 126401 (2009).

[15] P. Werner and A. J. Millis, Phys. Rev. Lett. 99, 126405 (2007).

[16] R. H. Zadik, Y. Takabayashi, G. Klupp, R. H. Colman, A. Y. Ganin, A. Potočnik, P. Jeglič, D. Arčon, P. Matus, K. Kamarás, Y. Kasahara, Y. Iwasa, A. N. Fitch, Y. Ohishi, G. Garbarino, K. Kato, M. J. Rosseinsky, and K. Prassides, Sci. Adv. 1, e1500059 (2015).

[17] S. Hoshino and P. Werner, Phys. Rev. Lett. 118, 177002 (2017).

[18] W. Metzner and D. Vollhardt, Phys. Rev. Lett. 62, 324 (1989).

[19] A. Georges, G. Kotliar, W. Krauth, and M. J. Rozenberg, Rev. Mod. Phys. 68, 13 (1996).

[20] T. Pruschke, M. Jarrell, and J. Freericks, Adv. Phys. 44, 187 (1995).

[21] P. Werner, A. Comanac, L. de' Medici, M. Troyer, and A. J. Millis, Phys. Rev. Lett. 97, 076405 (2006).

[22] E. Gull, A. J. Millis, A. I. Lichtenstein, A. N. Rubtsov, M. Troyer, and P. Werner, Rev. Mod. Phys. 83, 349 (2011).

[23] Y. Nomura and R. Arita, Phys. Rev. B 92, 245108 (2015).

[24] Y. Nomura, K. Nakamura, and R. Arita, Phys. Rev. B 85, 155452 (2012).

[25] M. Fabrizio and E. Tosatti, Phys. Rev. B 55, 13465 (1997).

[26] M. Capone, M. Fabrizio, C. Castellani, and E. Tosatti, Science 296, 2364 (2002).

[27] Y. Nomura, S. Sakai, M. Capone, and R. Arita, Sci. Adv. 1, e1500568 (2015).

[28] G. Kotliar and H. Kajueter, Phys. Rev. B 54, R14221 (1996).

[29] M. J. Rozenberg, Phys. Rev. B 55, R4855 (1997).
[30] K. Held and D. Vollhardt, Eur. Phys. J. B 5, 473 (1998).

[31] J. E. Han, M. Jarrell, and D. L. Cox, Phys. Rev. B 58, R4199 (1998).

[32] Y. Imai and N. Kawakami, J. Phys. Soc. Jpn. 70, 2365 (2001).

[33] A. Koga, Y. Imai, and N. Kawakami, Phys. Rev. B 66, 165107 (2002).

[34] A. Koga, T. Ohashi, Y. Imai, S.-I. Suga, and N. Kawakami, J. Phys. Soc. Jpn. 72, 1306 (2003).

[35] K. Inaba, A. Koga, S.-i. Suga, and N. Kawakami, Phys. Rev. B 72, 085112 (2005).

[36] A. M. Läuchli and P. Werner, Phys. Rev. B 80, 235117 (2009).

[37] T. Momoi and K. Kubo, Phys. Rev. B 58, R567 (1998).

[38] H. Yanatori and A. Koga, Phys. Rev. B 94, 041110 (2016).

[39] A. Golubeva, A. Sotnikov, A. Cichy, J. Kuneš, and W. Hofstetter, Phys. Rev. B 95, 125108 (2017).

[40] A. Koga and P. Werner, Phys. Rev. B 91, 085108 (2015).

[41] A. Koga and H. Yanatori, J. Phys. Soc. Jpn. 86, 034702 (2017).

[42] A. Georges, G. Kotliar, and W. Krauth, Z. Phys. B 92, 313 (1993).

[43] A. Y. Ganin, Y. Takabayashi, P. Jeglič, D. Arčon, A. Potočnik, P. J. Baker, Y. Ohishi, M. T. McDonald, M. D. Tzirakis, A. McLennan, G. R. Darling, M. Takata, M. J. Rosseinsky, and K. Prassides, Nature (London) 466, 221 (2010).

[44] T. Misawa and M. Imada, arXiv:1711.10205.

[45] E. G. C. P. van Loon, A. I. Lichtenstein, M. I. Katsnelson, O. Parcollet, and H. Hafermann, Phys. Rev. B 90, 235135 (2014).

[46] A. Koga and P. Werner, J. Phys. Soc. Jpn. 79, 064401 (2010).

[47] A. Koga and P. Werner, Phys. Rev. A 84, 023638 (2011).

[48] B. Bauer, L. D. Carr, H. G. Evertz, A. Feiguin, J. Freire, S. Fuchs, L. Gamper, J. Gukelberger, E. Gull, S. Guertler, A. Hehn, R. Igarashi, S. V. Isakov, D. Koop, P. N. Ma, P. Mates, H. Matsuo, O. Parcollet, G. P. owski, J. D. Picon, L. Pollet, E. Santos, V. W. Scarola, U. Schollwöck, C. Silva, B. Surer, S. Todo, S. Trebst, M. Troyer, M. L. Wall, P. Werner, and S. Wessel, J. Stat. Mech. (2011) P05001. 